\title{
A Retrospective Medical Record Review of Utilization Patterns and Medical Resource Use Associated with Repository Corticotropin Injection among Patients with Rheumatologic Diseases in the United States
}

\author{
Winnie W. Nelson (ID - Michael J. Philbin · Jack R. Gallagher • \\ Kylee Heap · Susan Carroll · George J. Wan \\ Received: September 11, 2017 / Published online: October 25, 2017 \\ (C) The Author(s) 2017. This article is an open access publication
}

\section{ABSTRACT}

Introduction: Repository corticotropin injection (RCI) has anti-inflammatory and immune-modulatory effects and is approved for multiple indications, including several rheumatologic conditions. The aims of this nationally representative, retrospective, observational study were to describe patient characteristics, RCI treatment patterns, and barriers to RCI use in patients with rheumatologic disease, and to compare medical resource use (MRU) before and after RCI therapy.

Methods: A random sample of US physicians was recruited to abstract the medical records of deidentified patients with a diagnosis of rheumatoid arthritis (RA), psoriatic arthritis (PsA), dermatomyositis/polymyositis (DM/PM), or systemic lupus erythematosus (SLE) who had been treated with RCI in the previous 24 months. Patient characteristics and patterns of RCI use were identified. Mean MRU in the 3 months before and after RCI therapy was compared using paired-samples $t$ tests.

Enhanced content To view enhanced content for this article go to http://www.medengine.com/Redeem/09CC F0600AC3FE19.

W. W. Nelson $(\varangle) \cdot$ M. J. Philbin · G. J. Wan

Mallinckrodt Pharmaceuticals, Bedminster, NJ, USA

e-mail: Winnie.Nelson@mallinckrodt.com

J. R. Gallagher · K. Heap · S. Carroll

Clarity Pharma Research LLC, Spartanburg, SC, USA
Results: A total of 449 physicians abstracted the medical records of 217 RA, 190 PsA, 254 $\mathrm{DM} / \mathrm{PM}$, and 95 SLE patients. In all groups combined, patients had received a mean of 3.3 treatment medications before initiating RCI. Most patients (75\%-94\%) were receiving RCI for the first time, indicating that repeated courses of RCI were uncommon. RCI was used as bridge therapy in $18 \%$ of patients. Approximately $24 \%$ of patients encountered an obstacle in accessing RCI, primarily insurance-related. After RCI therapy, the number of hospitalizations and hospital days were significantly reduced for all cohorts (all $P<0.05$ ), and the number of outpatient visits was significantly lower for all cohorts $(P<0.05)$ except the SLE cohort $(P=0.3230)$. Study limitations include potentially incomplete data in the medical records and a relatively short duration for capturing MRU changes.

Conclusions: RCI was used primarily as lateline therapy in patients with rheumatologic diseases. Medical resource use was significantly lower in the 3 months after therapy compared with 3 months prior. This finding suggests that RCI may improve disease control and warrants further evaluation.

Funding: Mallinckrodt Pharmaceuticals.

Keywords: ACTH; H.P. Acthar $^{\circledR}$ Gel; Dermatomyositis; Drug therapy; Health care utilization; Polymyositis; Psoriatic arthritis; 
Repository corticotropin injection; RCI; Rheumatoid arthritis; Systemic lupus erythematosus

\section{INTRODUCTION}

Repository corticotropin injection (RCI; H. P. Acthar ${ }^{\circledR}$ Gel, Mallinckrodt Pharmaceuticals, Hazelwood, MO) is a highly purified, prolonged-release adrenocorticotropic hormone (ACTH) analog with anti-inflammatory and immunomodulatory properties $[1,2]$. It was first approved in 1952 and is currently approved for multiple indications, including as an adjunctive therapy for short-term administration (to tide the patient over an acute episode or exacerbation) in the treatment of rheumatoid arthritis (RA) and psoriatic arthritis (PsA) as well as during an exacerbation or as maintenance therapy in systemic dermatomyositis/polymyositis (DM/PM) and systemic lupus erythematosus (SLE) [1].

Rheumatologic diseases have a substantial impact on quality of life and require ongoing management. Pharmacotherapy is an essential component of both maintenance therapy and management of exacerbations. Recent progress in understanding the effects of RCI in modulating immune responses has led to increased interest in RCI as a therapeutic choice [2]. Clinical trials and retrospective case series have found RCI to be effective for reducing disease activity and signs/symptoms as well as improving functional status in patients with RA, PsA, DM/PM, and SLE [3-12]. Claims data studies that examined medical resource use (MRU) relating to rheumatologic disorders reported that RCI treatment was associated with reduced health care utilization $[13,14]$. In addition, a study of patients with RA, DM/PM, or SLE found reduced use of corticosteroids and biologics after RCI therapy [15].

Additional real-world data are needed to better understand patterns of RCI use, barriers to use, and the potential impact of RCI therapy on MRU in patients with rheumatologic diseases. The aims of this study were to describe patient characteristics, RCI treatment patterns, and barriers to RCI use in patients with RA, PsA,
DM/PM, or SLE, and to compare MRU before and after RCI treatment in a large patient population.

\section{METHODS}

This was a national, retrospective, observational study of patients with rheumatologic disease (RA, PsA, DM/PM, or SLE) who had received RCI therapy. To obtain a nationally representative sample of patients, we recruited physicians in eight medical specialty areas (cardiology, dermatology, internal medicine, nephrology, neurology, primary care/family practice/general practice, pulmonology, and rheumatology) using the American Medical Association (AMA) Physician Masterfile. This file contains current data for more than 1.4 million physicians, residents, and medical students in the United States and is updated continuously by the AMA. Randomly selected physicians in the targeted specialties were contacted via email or telephone and screened for eligibility. Eligible physicians were those with at least one patient who had received RCI to treat a condition of interest in the previous 12 months.

Patient inclusion criteria were (1) a diagnosis of RA, PsA, DM/PM, or SLE, and (2) completion of at least one course of RCI therapy in the previous 24 months and at least 3 months before medical record review. Participating physicians were instructed to select patients for the study in the order of last seen-a randomization technique. The order of last seen does not imply newness of diagnosis but rather recency of a patient's chart being updated via an office visit. Physicians with patients in only one target disease area were asked to provide up to four cases; those with patients in two or more target disease areas could provide up to six cases.

Data collection took place from February 2016 to April 2016. Physicians or their designated staff abstracted patient medical record data using an electronic data collection instrument. The data collection instrument was pretested by qualified physicians with qualified patient charts. Abstracted data included patient demographic information, diagnoses, 
medications, number of RCI therapy courses, RCI dose, reason for RCI use, and barriers to RCI use as well as number of hospital admissions, number of hospital days, and number of outpatient visits in the 3 months before and after RCI initiation. To ensure data accuracy, technical support was made available to physicians and the data collection software was programmed to automatically calculate date ranges, dosing conversions, and other calculated values. Physicians were alerted when a data entry was out of range and asked to verify or correct the information. To protect patient and physician confidentiality, all data were deidentified at the time of data collection and reported in the aggregate. This article is based on previously existing observational data, and the research did not involve any new interventional studies of human or animal subjects performed by any of the authors. This retrospective study used deidentified data, and no personal health information was collected. For this type of study, formal consent is not required. The study was classified as exempt by Solutions Institutional Review Board (Little Rock, AR, USA).

\section{Statistical Analysis}

To ensure that each patient's probability of being selected for the study was appropriately represented, the data were weighted to correct for over- or underrepresentation of particular study segments in the study sample. Patient characteristics were assessed using descriptive statistics. For comparisons of interval-scale data, analysis of variance tests or $t$ tests were used. For categorical data comparisons, chi-square tests of independence and $z$ tests for column proportions were used. Medical resource use was defined as the mean number of all-cause hospital admissions, hospital days, and outpatient visits. Paired-sample $t$ tests were used to compare MRU in the 3 months before and after RCI use. The 0.05 level was used to determine statistical significance. SPSS Statistics for Windows version 22.0 (IBM Corporation, Armonk, NY, USA) was used for data tabulation, to develop statistical weights, and for significance testing.

\section{RESULTS}

A total of 449 physicians participated in the study, of whom $35 \%$ were rheumatologists, $25 \%$ neurologists, $13 \%$ internists, $9 \%$ dermatologists, $6 \%$ nephrologists, $6 \%$ pulmonologists, $5 \%$ primary care physicians, and $1 \%$ cardiologists. Data were obtained for 217 patients with RA, 190 patients with PsA, 254 patients with DM/ PM, and 95 patients with SLE. Table 1 presents the demographic and clinical characteristics of the study patients with rheumatologic conditions who were receiving RCI. Mean age (standard deviation [SD]) in years was 51.69 (11.94) for the RA group, 51.96 (12.32) for the PsA group, 50.26 (12.83) for the DM/PM group, and 48.46 (10.95) for the SLE group. The groups ranged from $52 \%$ to $79 \%$ female, with the SLE group having a significantly higher proportion of females. In the RA, PsA, and DM/PM groups, approximately one-half to two-thirds of patients were Caucasian/non-Hispanic. As expected, the SLE group had a significantly higher proportion of African Americans (48\%) than did the other groups. The most common comorbidities were hypertension, hyperlipidemia, gastrointestinal disorders, diabetes, and mood disorders. Across groups, more than one-third of patients had a diagnosis of hypertension and more than one-fifth had a diagnosis of hyperlipidemia. Between $22 \%$ and $36 \%$ of patients had no comorbidities.

\section{RCI Treatment Patterns and Barriers to Access}

In the four groups combined, a mean of 3.3 medications had been used before initiation of RCI therapy (3.6, 3.3, 2.9, and 3.6 medications in the RA, PsA, DM/PM, and SLE groups, respectively). Most RCI regimens (75\%-94\%) were the first-time use of RCI for the patient (Table 2). In all groups combined, $17 \%$ of patients received RCI as a bridge to new therapy $(15 \%, 19 \%, 21 \%$, and $5 \%$ of the RA, PsA, DM/ PM, and SLE groups, respectively).

Among those medical records with information about medication access, about one-fourth (24\%) of patients experienced one or more 
Table 1 Demographic and clinical characteristics of study patients with a rheumatologic condition receiving repository corticotropin injection

\begin{tabular}{|c|c|c|c|c|}
\hline Characteristic & $\mathrm{RA}(n=217)$ & PsA $(n=190)$ & $\mathrm{DM} / \mathrm{PM}(n=254)$ & SLE $(n=95)$ \\
\hline Mean age $(\mathrm{SD})$ in years & $51.69(11.94)$ & $51.96(12.32)$ & $50.26(12.83)$ & $48.46(10.95)$ \\
\hline \multicolumn{5}{|l|}{ Age category (\%) } \\
\hline $6-21$ years & 0 & 2 & 5 & 6 \\
\hline $22-35$ years & 10 & 7 & 10 & 10 \\
\hline $36-50$ years & 32 & 38 & 37 & 42 \\
\hline $51-64$ years & 44 & 37 & 35 & 29 \\
\hline $65+$ years & 14 & 16 & 13 & 13 \\
\hline \multicolumn{5}{|l|}{ Gender (\%) } \\
\hline Male & 35 & 48 & 47 & 21 \\
\hline Female & 65 & 52 & 53 & 79 \\
\hline \multicolumn{5}{|l|}{ Ethnicity (\%) } \\
\hline African American & 23 & 15 & 24 & 48 \\
\hline American Indian/Alaska Native & 0 & 2 & 5 & 4 \\
\hline Asian & 4 & 9 & 6 & 6 \\
\hline Caucasian/non-Hispanic & 62 & 66 & 54 & 36 \\
\hline Hispanic/Latino & 11 & 8 & 11 & 5 \\
\hline Native Hawaiian/Pacific Islander & 0 & 0 & 0 & 1 \\
\hline \multicolumn{5}{|l|}{ Comorbidities (\%) } \\
\hline Hypertension & 32 & 33 & 32 & 35 \\
\hline Hyperlipidemia & 23 & 29 & 24 & 21 \\
\hline Gastrointestinal disorders & 19 & 11 & 10 & 9 \\
\hline Diabetes & 18 & 17 & 23 & 21 \\
\hline Mood disorders & 14 & 16 & 10 & 12 \\
\hline Kidney and urologic disorders & 6 & 5 & 7 & 14 \\
\hline Heart conditions & 4 & 7 & 9 & 3 \\
\hline Cancer & 3 & 7 & 4 & 6 \\
\hline Metabolic disorders & 3 & 3 & 4 & 5 \\
\hline Hearing problems & 3 & 4 & 5 & 5 \\
\hline Respiratory conditions & 2 & 1 & 4 & 7 \\
\hline Gout & 2 & 7 & 7 & 7 \\
\hline Other CNS conditions & 1 & 0 & - & - \\
\hline Other & 1 & 0 & 1 & - \\
\hline
\end{tabular}


Table 1 continued

\begin{tabular}{lllll}
\hline Characteristic & RA $(n=217)$ & PsA $(n=190)$ & DM/PM $(n=254)$ & SLE $(n=95)$ \\
\hline Vision loss & 0 & 1 & 1 & 0 \\
No comorbid conditions & 36 & 30 & 25 & 22 \\
\hline
\end{tabular}

$C N S$ central nervous system, $D M / P M$ dermatomyositis/polymyositis, $P s A$ psoriatic arthritis, $R A$ rheumatoid arthritis, $S D$ standard deviation, SLE systemic lupus erythematosus

Table 2 Repository corticotropin injection regimens in patients with rheumatologic disease

\begin{tabular}{lllll}
\hline RCI use & \multicolumn{2}{l}{ Percentage of regimens } & & \\
\cline { 2 - 5 } & RA (\%) & PsA (\%) & DM/PM (\%) & SLE (\%) \\
\hline First-time use & 92 & 85 & 75 & 94 \\
Second-time use & 7 & 13 & 16 & 4 \\
Third-time use & 1 & 2 & 9 & 1 \\
Fourth-time use & 0 & 0 & 0 & 1 \\
\hline
\end{tabular}

$D M / P M$ dermatomyositis/polymyositis, $P s A$ psoriatic arthritis, $R A$ rheumatoid arthritis, $R C I$ repository corticotropin injection, SLE systemic lupus erythematosus

obstacles to obtaining RCI (23\% of RA patients, $29 \%$ of PsA patients, $26 \%$ of DM/PM patients, and $14 \%$ of SLE patients). Among this subset of patients, the most common obstacles were the need for prior authorization (60\% of patients) and high out-of-pocket costs (53\% of patients). Lack of insurance, inadequate insurance, and formulary-related obstacles were other common barriers (Table 3).

\section{Medical Resource Use}

Mean medical resource use in the 3 months before and after RCI therapy is shown in

Table 3 Obstacles to repository corticotropin injection access in patients encountering one or more obstacles

\begin{tabular}{lllll}
\hline Obstacle & \multicolumn{3}{l}{ Percentage (\%) of patients } \\
\cline { 2 - 5 } & $\mathbf{R A}(\boldsymbol{n}=\mathbf{3 7})^{\mathbf{a}}$ & PsA $(\boldsymbol{n}=\mathbf{3 3})^{\mathbf{a}}$ & $\mathbf{D M} / \mathbf{P M}(\boldsymbol{n}=\mathbf{4 7})^{\mathbf{a}}$ & SLE $(\boldsymbol{n}=\mathbf{9})^{\mathbf{a}, \mathbf{b}}$ \\
\hline Prior authorization & 61 & 71 & 51 & 55 \\
High out-of-pocket cost & 52 & 61 & 47 & 62 \\
Inadequate insurance coverage & 30 & 29 & 28 & 63 \\
Formulary & 39 & 27 & 25 & 21 \\
Lack of insurance coverage & 20 & 34 & 34 & 11 \\
\hline
\end{tabular}

$D M / P M$ dermatomyositis/polymyositis, $P s A$ psoriatic arthritis, $R A$ rheumatoid arthritis, $R C I$ repository corticotropin injection, SLE systemic lupus erythematosus

a The subset of patients in the cohort whose medical records indicated an obstacle to drug access. Some patients had more than one diagnosis of interest and are counted in more than one cohort

b Caution, small $n$ 
Table 4 Comparison of medical resource use in the 3 months before and after repository corticotropin injection treatment

\begin{tabular}{|c|c|c|c|c|c|c|c|}
\hline \multirow[t]{2}{*}{ Resource use } & \multirow[t]{2}{*}{ Condition $^{\mathrm{a}}$} & \multicolumn{2}{|c|}{ Mean number per patient } & \multirow[t]{2}{*}{ Mean difference } & \multirow[t]{2}{*}{ SD } & \multicolumn{2}{|l|}{ 95\% CI } \\
\hline & & Pre-RCI & Post- RCI & & & Lower & Upper \\
\hline \multirow[t]{4}{*}{ Hospital admissions (count) } & RA & 0.07 & 0.02 & $-0.053^{*}$ & 0.373 & -0.103 & -0.003 \\
\hline & PsA & 0.24 & 0.16 & $-0.079^{*}$ & 0.334 & -0.127 & -0.031 \\
\hline & $\mathrm{DM} / \mathrm{PM}$ & 0.46 & 0.19 & $-0.271^{*}$ & 0.672 & -0.356 & -0.187 \\
\hline & SLE & 0.17 & 0.03 & $-0.137^{*}$ & 0.426 & -0.224 & -0.05 \\
\hline \multirow[t]{4}{*}{ Hospital days (days) } & RA & 0.23 & 0.04 & $-0.191^{*}$ & 1.405 & -0.379 & -0.003 \\
\hline & PsA & 0.37 & 0.15 & $-0.222^{*}$ & 0.984 & -0.363 & -0.08 \\
\hline & $\mathrm{DM} / \mathrm{PM}$ & 1.42 & 0.35 & $-1.064^{*}$ & 3.181 & -1.465 & -0.664 \\
\hline & SLE & 0.45 & 0.04 & $-0.41^{*}$ & 1.64 & -0.745 & -0.075 \\
\hline \multirow[t]{4}{*}{ Outpatient visits (count) } & RA & 2.80 & 2.38 & $-0.426^{*}$ & 1.306 & -0.6 & -0.251 \\
\hline & PsA & 3.18 & 2.46 & $-0.72^{*}$ & 1.46 & -0.93 & -0.51 \\
\hline & $\mathrm{DM} / \mathrm{PM}$ & 2.72 & 2.01 & $-0.71^{*}$ & 1.791 & -0.936 & -0.485 \\
\hline & SLE & 2.74 & 2.55 & -0.188 & 1.843 & -0.564 & 0.188 \\
\hline
\end{tabular}

$C I$ confidence interval, $D M / P M$ dermatomyositis/polymyositis, $P s A$ psoriatic arthritis, $R A$ rheumatoid arthritis, $R C I$ repository corticotropin injection, $S D$ standard deviation, $S L E$ systemic lupus erythematosus

${ }^{*} P<0.05$

${ }^{a} \mathrm{RA}, n=217$; PsA, $n=188$; DM/PM, $n=245$; SLE, $n=95$ (number of patients differs from overall counts because of missing data for 2 PsA and $9 \mathrm{DM} / \mathrm{PM}$ patients)

Table 4. After RCI therapy, the mean number of hospital admissions and hospital days was lower in all groups and the mean number of outpatient visits was lower in the RA, PsA, and DM/ PM groups. The RA and SLE groups experienced the largest percent decreases in hospital admissions $(71 \%$ and $82 \%$, respectively; both $P<0.05)$ and number of hospital days (83\% and 91\%, respectively; both $P<0.05$ ) (Fig. 1). The $\mathrm{DM} / \mathrm{PM}$ and PsA groups experienced the largest decreases in number of outpatient visits $(26 \%$ and $23 \%$, respectively, both $P<0.05$ ). Although the SLE group experienced a $7 \%$ decrease in the number of outpatient visits after RCI therapy, this difference was not statistically significant $(P=0.323)$.

\section{DISCUSSION}

In this nationally representative, retrospective, observational study of patients with rheumatologic disease, we found that RCI was primarily used as late-line therapy, with patients receiving an average of 3.3 rheumatologic drugs before the initiation of RCI. Most RCI treatment courses represented the first-ever use of the agent, indicating that repeated use of RCI was uncommon. The groups were generally similar in terms of demographic and clinical characteristics, except that the SLE group had significantly higher percentages of females and African Americans, as expected. This difference reflects national epidemiological data for SLE, which indicate that $90 \%$ of people with lupus are female and African American women are three times more likely than Caucasian women to develop the disease [16]. Although RCI is indicated for the management of rheumatologic disease exacerbations or as maintenance therapy, we found that about 1 in 6 RCI regimens was initiated as a bridge to a new therapy. Of note, approximately 1 in 4 patients encountered barriers to RCI access. The PsA and 


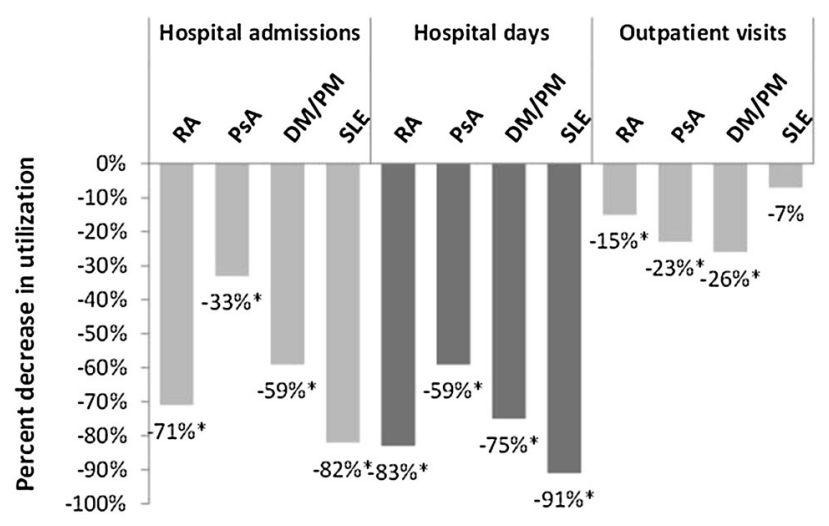

Fig. 1 Percent decrease in medical resource use after repository corticotropin injection treatment in patients with rheumatologic disease. $D M / P M$ dermatomyositis/ polymyositis, Ps $A$ psoriatic arthritis, $R A$ rheumatoid arthritis, SLE systemic lupus erythematosus. Values reflect

$\mathrm{DM} / \mathrm{PM}$ groups were most likely to encounter barriers (29\% and $26 \%$ of patients, respectively). Most obstacles were insurance-related, such as prior authorization requirements (60\%) and high out-of-pocket costs (53\%). In comparing MRU before and after RCI therapy, we observed $33 \%-82 \%$ fewer hospital admissions and $59 \%-91 \%$ fewer hospital days in all four cohorts, with the SLE cohort having the largest reductions. For outpatient visits, we observed $15 \%-26 \%$ fewer outpatient visits in three cohorts (RA, PsA, and DM/PM) after therapy, whereas the SLE cohort had a 7\% reduction that was not statistically significant.

To our knowledge, this is the first study to report contemporary real-world data for RCI treatment in a large national sample of patients with rheumatologic disorders. Importantly, the study included patients who had used multiple prior therapies. These patients are likely to be difficult to treat, and a better understanding of their therapeutic options is sorely needed. The finding that RCI is associated with reduced MRU across four rheumatologic diseases is a possible indication of improved disease control and may inform new and effective approaches to disease management.

Previous studies have reported that treatment of rheumatologic disease with RCI is associated with reductions in health care utilization. A study of RCI use in 29 patients with the percent change in health care encounters (number of hospitalizations, number of hospital days, and number of outpatient visits) in the 3 months after RCI treatment compared with the 3 months before treatment. ${ }^{*} P<0.05$

SLE reported a decrease in the mean number of all-cause outpatient visits per patient-year after treatment and a decrease in SLE-related medical costs from $\$ 3011$ to $\$ 893$ PPPM [14]. In a sample of 180 patients with RA, all-cause hospital admissions decreased from 42 to 25 per 1000 patient-years and RA-related hospital admissions decreased from 13 to 4 per 1000 patientyears after RCI therapy [14]. The number of all-cause and RA-related outpatient visits per patient-year also decreased after RCI therapy, as did the number of all-cause and RA-related emergency department visits per 1000 patient-years. In addition, all-cause and RA-related medical costs were correspondingly lower after RCI therapy (all-cause, $\$ 1881$ vs. $\$ 682$ PPPM; RA-related, \$658 vs. \$93 PPPM), driven primarily by lower inpatient costs. In a study of RCI versus intravenous immunoglobulin or rituximab in 1180 patients with DM/PM, RCI was associated with fewer hospital admissions and outpatient visits after therapy than intravenous immunoglobulin, and with fewer outpatient visits than rituximab [13]. The reduced health care utilization after RCI therapy observed in our study and in other studies suggests that patients who receive RCI have improved disease control, and warrants additional research.

Our study is not unique in finding that many patients experience obstacles to obtaining 
prescribed medication. Barriers to accessing medications are a significant and growing problem nationally, even in insured populations. The Commonwealth Fund reported that $23.1 \%$ of the US population reports not filling a prescription or skipping a dose in the last 12 months because of cost [17]. An analysis of the care of patients with RA found that patients covered by Medicaid were less likely than those covered by private insurance to receive disease-modifying antirheumatic drugs, and they were also more likely to experience a delay in obtaining medications [18]. This disparity in medication access was associated with a greater likelihood of cognitive, social, and physical limitations and lower general health and quality of life. In our study, insurance-related problems, including the need for prior authorization, high out-of-pocket costs, and inadequate insurance coverage, were the most common obstacles to RCI access. Considering that RCI is often prescribed for difficult-to-treat patients who have already tried several other therapies, these obstacles to timely medication access are of particular concern.

Study limitations include the possibility that medical records were incomplete or missing data. In addition, data errors might have occurred during medical record abstraction. Several measures were employed to ensure data accuracy, including the use of software with automatic calculation functions and error alerts and a review of outliers by the research team. Data regarding concomitant therapies and disease activity, which would provide additional context for understanding the role of RCI therapy, were not collected. Another potential limitation is an insufficient duration of follow-up for capturing changes in MRU. A significant strength of the study is the use of a large nationally representative patient sample that can be generalized to the US population of patients with rheumatologic conditions.

\section{CONCLUSION}

In this large sample of patients with rheumatologic disease, RCI was used primarily as lateline therapy. Most RCI treatment courses represented the first-ever use of the agent, indicating that repeated use of RCI was uncommon. Approximately 1 in 4 patients experienced a barrier to medication access, particularly PsA and DM/PM patients. Treatment with RCI was associated with significantly fewer hospital admissions and hospital days in the 3 months after therapy. All cohorts except for the SLE cohort also experienced significant reductions in the number of outpatient visits after RCI treatment. The finding of a reduced MRU suggests that RCI may improve disease control. Its effectiveness for difficult-to-treat patients with RA, PsA, DM/PM, and SLE warrants further evaluation.

\section{ACKNOWLEDGEMENTS}

The authors thank the physicians who participated in this study. Sponsorship of this study and the paper's article processing charges were funded by Mallinckrodt Pharmaceuticals, Bedminster, NJ, USA. Writing and editorial assistance was provided by Linda Henry, Judith Hurley, and Esther Tazartes of Global Outcomes Group, Reston, VA, USA, and was funded by Mallinckrodt Pharmaceuticals, Bedminster, NJ, USA. All named authors meet the International Committee of Medical Journal Editors (ICMJE) criteria for authorship for this manuscript, take responsibility for the integrity of the work as a whole, and have given final approval for the version to be published. All authors had full access to all of the data in this study and take responsibility for the integrity of the data and accuracy of the data analysis.

Disclosures. Winnie W. Nelson is an employee of Mallinckrodt Pharmaceuticals, Bedminster, NJ, USA, and declares that she has no other conflicts of interest. George J. Wan is an employee of Mallinckrodt Pharmaceuticals, Bedminster, NJ, USA, and declares that he has no other conflicts of interest. Michael J. Philbin was an employee of Mallinckrodt at the time of this study and declares that he has no other conflicts of interest. Jack R. Gallagher is an employee of Clarity Pharma Research LLC, 
Spartanburg, NC, USA, and declares that he has no conflicts of interest. Kylee Heap is an employee of Clarity Pharma Research LLC, Spartanburg, NC, USA, and declares that he has no conflicts of interest. Susan Carroll is an employee of Clarity Pharma Research LLC, Spartanburg, NC, USA, and declares that he has no conflicts of interest.

Compliance with Ethics Guidelines. This article is based on previously existing observational data, and the research did not involve any new interventional studies of human or animal subjects performed by any of the authors. This retrospective study used deidentified data, and no personal health information was collected. For this type of study, formal consent is not required. This study was classified as exempt by Solutions Institutional Review Board (Little Rock, AR, USA).

Data Availability. The datasets during and/ or analyzed during the current study are available from the corresponding author on reasonable request.

Open Access. This article is distributed under the terms of the Creative Commons Attribution-NonCommercial 4.0 International License (http://creativecommons.org/licenses/by-nc/4. $0 /$ ), which permits any noncommercial use, distribution, and reproduction in any medium, provided you give appropriate credit to the original author(s) and the source, provide a link to the Creative Commons license, and indicate if changes were made.

\section{REFERENCES}

1. Acthar HP. Gel (repository corticotropin injection) [prescribing information]. Hazelwood: Mallinckrodt ARD; 2015.

2. Berkovich R, Agius MA. Mechanisms of action of ACTH in the management of relapsing forms of multiple sclerosis. Ther Adv Neurol Disord. 2014;7:83-96.

3. Aggarwal R, Marder G, Loganathan P, et al. Efficacy and safety of adrenocorticotropic hormone gel
(Acthar Gel ${ }^{\circledR}$ ) in refractory dermatomyositis or polymyositis [ACR/ARHP abstract 2363]. Arthritis Rheumatol. 2015;67(10):2848-9.

4. Fiechtner JJ, Montroy T. Six months' treatment of moderately to severely active systemic lupus erythematosus with repository corticotropin injection: an extension of a single-site, open-label trial. J Immunol Clin Res. 2016;3:1025-31.

5. Furie R, Mitrane M, Zhao E, Das M, Li D, Becker PM. Efficacy and tolerability of repository corticotropin injection in patients with persistently active SLE: results of a phase 4 , randomized, controlled pilot study. Lupus Sci Med. 2016;3:e00180.

6. Gaylis N, Needell S, Sagliani J. The effect of adrenocorticotropin gel (HP Acthar Gel) in combination with MTX in newly diagnosed RA patients from a clinical and structural perspective. Ann Rheum Dis. 2015;74(2):10661067.

7. Gillis TM, Crane M, Hinkle C, Wei N. HP Acthar Gel (repository corticotropin injection) as adjunctive therapy in patients with rheumatoid arthritis who have failed at least three biologic therapies with different modes of action. Ann Rheum Dis. 2015;74(2):1066.

8. Levine T. Treating refractory dermatomyositis or polymyositis with adrenocorticotropic hormone gel: a retrospective case series. Drug Des Devel Ther. 2012;6:133-9.

9. Levine T, Malone J, Efthimiou P, et al. HP Acthar $\mathrm{Gel}^{\circledR}$ in dermatomyositis and polymyositis treatment registry: an interim analysis. J Neurol Dis. 2016;4(5):292.

10. Li X, Golubovsky J, Hui-Yuen J, et al. Adrenocorticotropic hormone gel in the treatment of systemic lupus erythematosus: a retrospective study of patients [version 2]. F1000 Res. 2016;4:1103-14.

11. Pegram SB. The efficacy and tolerability of H.P. Acthar $^{\circledR}$ Gel (repository corticotropin injection) for the treatment of systemic lupus erythematosus. Ann Rheum Dis. 2015;74(2):1077-8.

12. Brown AN. Repository corticotropin injection in patients with refractory psoriatic arthritis: a retrospective case series. Open Access Rheumatol. 2016;8:97-102.

13. Knight T, Bond C, Popelar B, et al. Medical resource utilization in dermatomyositis/polymyositis patients treated with repository corticotropin injection, intravenous immunoglobulin, and/or rituximab. Clinicoecon Outcomes Res. 2017;9:271-9.

14. Wu B, Deshpande G, Gu T, Popelar B, Philbin M. Wan GJ (2017) Demographics, treatment patterns, 
and healthcare utilization and costs of repository corticotropin injection in patients with systemic lupus erythematosis or rheumatoid arthritis. J Med Econ. 2017;10(1080/13696998):1362411 (Published online August).

15. Myung G, Nelson WW, McMahon MA (2017) Effects of repository corticotropin injection on medication use in patients with rheumatologic conditions: a claims data study. J Pharm Technol 2017;33:151-155.

16. Lupus Research Alliance. Who gets lupus. Lupus Research Alliance website. http://www.lupusny. org/about-lupus/who-gets-lupus. Accessed June 5, 2017

17. Morgan S, Kennedy J. Prescription drug accessibility and affordability in the United States and abroad. Issue Brief (Commonw Fund). 2010;89:1-12.

18. Cifaldi M, Renaud J, Ganguli A, Halpern MT. Disparities in care by insurance status for individuals with rheumatoid arthritis: analysis of the Medical Expenditure Panel Survey, 2006-2009. Curr Med Res Opin. 2016;32:2029-37. 